\title{
A Context-aware User-driven Strategy to Exploit Offloading and Sharing in Ultra-dense Deployments
}

\author{
F. Bouali, K. Moessner \\ 5G Innovation Centre (5GIC) \\ University of Surrey, UK \\ Email: \{f.bouali, k.moessner\}@ surrey.ac.uk
}

\author{
M. Fitch \\ BT Research \\ Adastral Park, Ipswich IP5 3RE, UK \\ Email: michael.fitch@bt.com
}

\begin{abstract}
This paper proposes a novel context-aware userdriven strategy to efficiently exploit all available bands and licensing regimes in ultra-dense deployments without prior knowledge about each combination. It relies first on fuzzy logic to estimate the suitability of each radio access technology (RAT) to support the requirements of various applications. Then, a fuzzy multiple attribute decision making (MADM) approach is developed to combine these estimates with the heterogeneous context components to assess the in-context suitability. Based on this metric, a spectrum management strategy is proposed to support interactive video sessions for a set of Bronze and Gold subscriptions. The results reveal that the proposed approach always assigns Gold users to the well-regulated licensed band, while switches Bronze users between licensed and unlicensed bands depending on the operating conditions. This results in a significant improvement of the quality-of-experience (QoE) compared to a baseline that exploits only licensed bands. Then, a comparative study is conducted between the available options to exploit unlicensed bands, namely Offloading and Sharing. The results show that the best option strongly depends on the existing load on WLAN. Therefore, a combined approach is proposed to efficiently switch between both options, which achieves the best QoE for all considered loads.
\end{abstract}

\section{Context/Motivation}

To relieve cellular networks of the ever-increasing load, small-cells operating in licensed bands have been deployed to extend capacity wherever needed [1]. Additionally, part of the traffic has been pushed to the existing WLANs in unlicensed bands, which is commonly referred to as Offloading [2]. After the recent unlicensed access granted to LTE, a new option has emerged, namely Sharing, where the small-cells directly share the unlicensed bands with WLANs. To meet the coexistence requirements introduced by the latter option, two candidates have been put forward. The first is a proprietary solution promoted by LTE-U Forum and referred to as LTEUnlicensed (LTE-U). It uses an adaptive ON/OFF duty cycle to co-exist with WLAN [3]. The second is the new licensedassisted access (LAA) standardized by 3GPP. It relies on a listen-before-talk (LBT) procedure to sense the medium and transmit only if it is idle [4]. While these candidates rely on a licensed anchor to provide robust connection management, muLTEfire has been recently proposed as a stand-alone LTEbased technology that operates only in unlicensed bands [5].

To efficiently exploit this multitude of connectivity options, many recent works have formulated an optimization problem that jointly considers licensed and unlicensed bands with the goal of maximizing sum-rate, energy and spectrum efficiency [6-8]. These works assume perfect knowledge of the channel state information (CSI) between the various smallcells, access points (APs) and surrounding user equipments (UEs), which is supposed to be collected in a central point.
However, such centralized approach may not be feasible for many practical scenarios e.g., fast-changing/mobile environments or when there is no collaboration at all between the available RATs. In such scenarios, a distributed decisionmaking that relies only on a limited amount of information would be much more practical. Another key limitation of these proposals is that they do not take into account many relevant components of the context (e.g., battery level and remaining balance), which may hurt the performance of some users e.g., a user with limited balance would get an "out-of-balance" drop after starting a high-quality video call over licensed, but may be able to finish the call with lower quality over a free unlicensed access. Finally, the target quality-of-service (QoS) level in the previous works was characterized solely in terms of a minimum bit-rate, which does not reflect the stringent requirements associated with multimedia applications.

The above discussion clearly call for a user-driven selection of the best available RAT depending on the specific operating conditions (e.g., interference and contention levels in licensed and unlicensed bands, respectively) and the various components of the context (e.g., QoS requirements, terminal capabilities and regulation rules). To this end, this paper exploits the generic framework previously built in [9] to enable a context-aware user-driven mode of operation. As an initial use case, the framework was applied in [9] to perform an intelligent offloading to WLAN for a mixture of best-effort and VoIP applications.

Motivated by the proven usefulness of the proposed framework in supporting spectrum management, this paper first extends it to support all emerging LTE-based candidates to access unlicensed bands (i.e., LTE-U, LAA and muLTEfire) together with their co-existence mechanisms (i.e., adaptive duty cycle and LBT). Based on this extension, a fuzzy MADM strategy that does not rely on any prior CSI knowledge is developed to exploit all available bands and licensing regimes in a given context. To cope with uncertainty, it first relies on fuzzy logic to estimate the out-of-context suitability level of each available RAT to support the requirements of various applications. Second, an MADM component combines these estimates with the heterogeneous components of the context to derive the in-context suitability levels. The proposed strategy is applied to efficiently exploit licensed and unlicensed bands to support interactive (i.e., delay-sensitive) video sessions in ultra-dense environments. In this respect, a methodology is developed to evaluate the quality-of-experience $(\mathrm{QoE})$ perceived by the end-users when actual video sequences are delivered. To the best of our knowledge, jointly exploiting licensed and unlicensed bands to meet the strict requirements associated with interactive video has not been considered previously. 


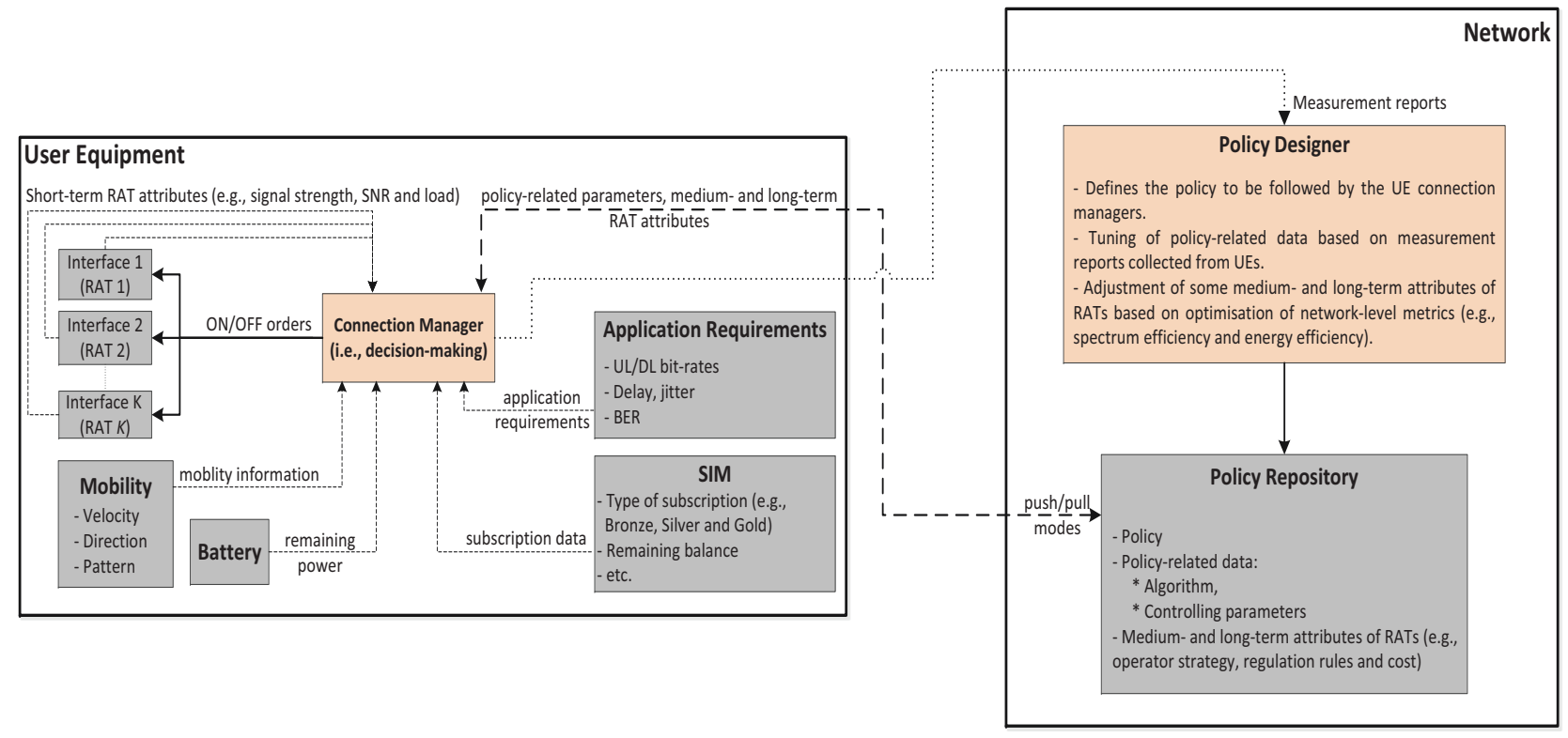

Fig. 1. Functional architecture for context-aware user-driven operation

The remainder of this paper is organized as follows. The considered framework to support spectrum management in ultra-dense environments is described in Section. II. Then, a fuzzy MADM strategy is developed in Section. III to perform a context-aware exploitation of all available RATs to support a set of heterogeneous applications. The initial results are presented in Section. IV, comparing the performance of various variants of the proposed approach to a baseline scheme that considers only licensed bands. The conclusions and future directions are provided in Section. V.

\section{CONTEXT-AWARE USER-DRIVEN FRAMEWORK FOR SPECTRUM MANAGEMENT in Ultra-DENSE ENVIRONMENTS}

\section{A. System model}

An ultra-dense environment is considered, where a set of $K$ available RATs $\left(\left\{R A T_{k}\right\}_{1 \leq k \leq K}\right)$ are exploited by various UEs to establish a set of $L$ applications $\left(\left\{A_{l}\right\}_{1 \leq l \leq L}\right)$. Each UE is assumed to belong to one of $S$ subscription profiles $\left(\left\{P_{s}\right\}_{1 \leq s \leq S}\right)$. Without loss of generality, $L=2$ applications are considered, namely interactive (i.e., delay-sensitive) video with stringent QoE requirements and FTP (i.e., delay-tolerant) transfer with loose QoS requirements, together with $S=2$ subscription profiles, namely Bronze and Gold with limited and unlimited balances, respectively.

To support the considered applications, a set of LTE smallcells are deployed on top of some existing WLAN APs. The various small-cells are assumed to be dual-access i.e., can jointly use licensed and unlicensed bands. Therefore, the following $K=3$ RATs are considered:

- Licensed/LTE: use the newly deployed small-cells to exploit licensed bands,

- Unlicensed/WLAN: offload part of the traffic to the existing WLANs,

- Unlicensed/LBT: use LTE small-cells to directly access and share unlicensed bands with the existing WLANs. To assess the unique potentials offered by unlicensed bands, a stand-alone muLTEfire RAT is assumed.
In the remainder of this paper, the band will be dropped from the notation for the sake of simplicity. Therefore, the above combinations will be denoted as LTE, WLAN and LBT, respectively.

\section{B. Target behavior}

Access to licensed bands (i.e., LTE) is assumed to be paid (i.e., consumes some units of the balance), while access to unlicensed bands (i.e., WLAN and LBT) is free of charge. The aim is to maximize usage of unlicensed bands provided that the application requirements are met. This means that the FTP transfer associated with loose QoS requirements can be always established on unlicensed bands, while interactive video sessions need to maximize usage of unlicensed bands as long as the associated requirements are met.

\section{Functional architecture}

To tackle the considered problem, the generic framework previously proposed in [9] will be instantiated. According to its functional architecture described in Fig. 1, a connection manager (CM) is introduced at the UE to implement a given decision-making policy. To this end, it exploits the relevant components of the context available locally (e.g., velocity and battery level) and a radio characterisation of each available RAT in terms of a set of short-term attributes (e.g., SNR and load) obtained e.g., through beacons and some medium- and long-term attributes (e.g., cost and regulation rules) stored in a policy repository together with all the policy-related data. The content of the policy repository may be retrieved in practice from a local instance following a pull or push mode using e.g., the Open Mobile Alliance-Device Management (OMA-DM) protocol [10]. To offer higher flexibility to the network manager, a policy designer entity builds and updates the policy repository content based on measurement reports collected from the various UEs and some potential networklevel constraints (e.g., operator strategy and regulation rules).

To achieve the target behavior set in Section. II-B, a fuzzy MADM implementation of the CM will be developed in the next section. 


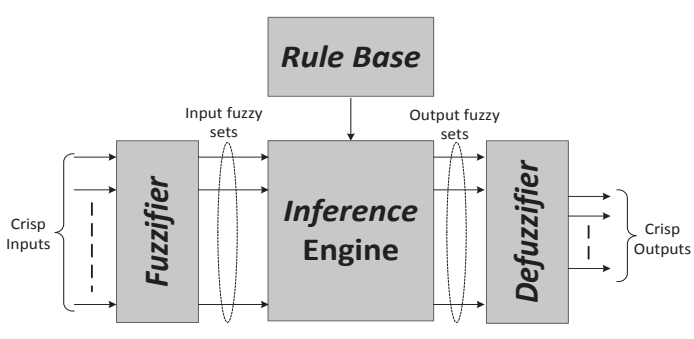

Fig. 2. Block diagram of the fuzzy logic controller

\section{Connection Manager: Fuzzy MADM DECISION-MAKING}

This section implements the CM of the functional architecture described in Fig. 1 to perform a context-aware exploitation of all available RATs to support each of the considered applications.

In accordance with the general guidelines given in [9], a three-step approach is proposed:

1) Design a fuzzy logic calculator to estimate the "out-ofcontext" suitability level of each $R A T_{k}$ to support the requirements of each application $A_{l}\left(s_{k, l}^{o c}\right)$ based on the available radio parameters.

2) Develop a fuzzy MADM methodology to combine $s_{k, l}^{o c}$ with the UE subscription profile (i.e., $P_{s}$ ) and all other components of the context to derive the so-named "incontext" suitability level $\left(s_{k, l}^{i c, s}\right)$.

3) Select the RAT that maximizes the in-context suitability level $s_{k, l}^{i c, s}$.

In what follows, each of the above steps will be implemented.

\section{A. Out-of-context suitability levels}

Given the uncertainty and lack of information associated with UEs, this section relies on fuzzy logic to estimate the suitability levels of each RAT to meet the requirements of the considered applications.

The key building block in fuzzy logic reasoning is the fuzzy logic controller (FLC) whose block diagram is described in Fig. 2 [11]. It is composed of three main stages, namely the fuzzifier, inference engine and defuzzifier. During fuzzification, crisp (i.e., real) input data are assigned a value between 0 and 1 corresponding to the degree of membership in a given fuzzy set. Then, the inference engine executes a set of if-then rules on the input fuzzy sets. These rules, referred to as inference rules, are maintained in a rule base that is typically built based on previous expert knowledge. Finally, the aggregated output fuzzy sets are converted into crisp outputs using a given defuzzification method.

In the following, a separate FLC is designed to estimate the suitability of each RAT to support the QoE requirements of the interactive video application.

1) LTE: The designed FLC and corresponding membership functions are described in Fig. 3. In particular, the following input parameters are considered:

- $R S R Q$ : the reference symbol received quality that captures the radio and interference conditions.

- T_Sched: the average time each packet received from upper layers waits before being scheduled. It reflects the load condition on the eNodeB and may be broadcasted in

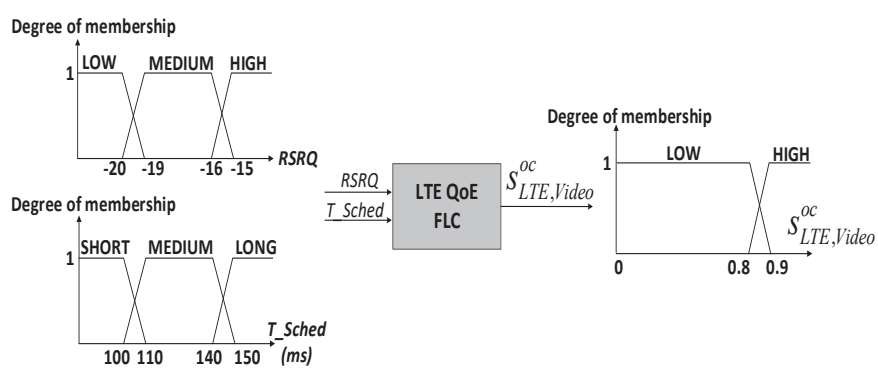

Fig. 3. LTE/Video FLC

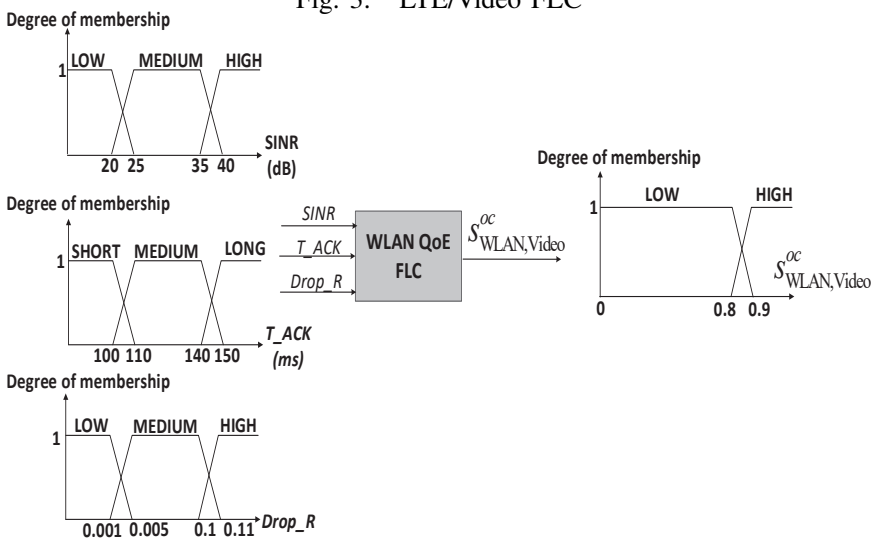

Fig. 4. WLAN/Video FLC

one of its system information blocks (SIBs). A non QoSaware scheduler (e.g., proportional fair (PF)) is initially assumed, which means that all packets are treated equally.

2) WLAN: The proposed FLC and associated membership functions are described in Fig. 4. Specifically, the set of input parameters is designed as follows:

- SINR: the signal-to-interference-and-noise-ratio of the AP beacon that reflects the radio and interference conditions.

- $T_{-} A C K$ : the average delay between receiving a packet from higher layers till successfully transmitting it (i.e., receiving the corresponding ACK from the receiver). It jointly captures the impact of the load served by the AP and channel contention (e.g., retransmissions due to collision). No 802.11e QoS support is considered initially, which means that all traffic types share the same $T_{-} A C K$.

- Drop_R: the rate of dropped packets. A packet is dropped whenever the MAC queue is full or the maximum retransmission limit is reached for an unacknowledged packet.

3) LBT: This option inherits some features (e.g., MAC scheduler) from LTE and others (e.g., contention-based access) from WLAN. In this respect, the designed FLC described in Fig. 5 is fed with the following inputs:

- RSRQ: the reference symbol received quality that captures the radio and interference conditions.

- T_Access: the average time each packet received from upper layers waits before being granted access to the channel. Compared to LTE (i.e., $T_{-}$Sched), it includes the additional delay (e.g., back-off) that may be introduced by the LBT procedure after a packet gets scheduled.

- NACK_R: the ratio of NACKs out of the HARQ-ACK feedback values available for the first sub-frame of the latest data burst. It reflects the likelihood of a collision as reflected by the rule recently selected by $3 \mathrm{GPP}$ to update the contention window size [12]. 


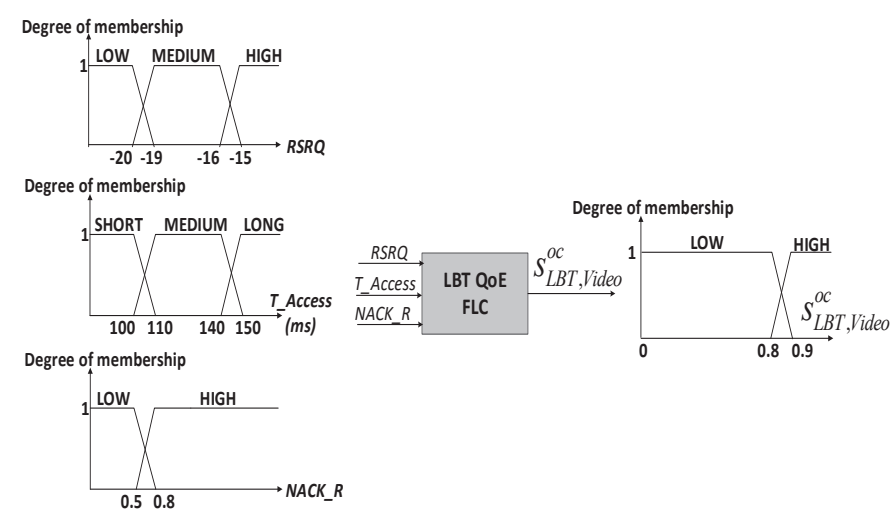

Fig. 5. LBT/Video FLC

For all FLCs, the inferences rules have been designed based on a sensitivity analysis to the various combinations of the input parameters, which is omitted for the sake of brevity. This mimics the adjustment performed by the policy designer of Fig. 1 based on the measurements reported by the UEs.

\section{B. In-context suitability levels}

In this section, the previous estimates are combined with the components of the context to derive the in-context suitability levels. To particularly cope with the heterogeneity of the context components, a methodology is developed based on multiple attribute decision making (MADM) [13].

In this respect, for each $k \in\{1, \ldots, K\}, R A T_{k}$ is characterized in terms of the following $M=4$ attributes:

- $s_{k, l}^{o c}$ : the out-of-context suitability to meet the application requirements. Recall that this is the output of the previous sub-section.

- cost $_{k}$ : the monetary cost of $R A T_{k}$.

- power $_{k}$ : the power consumption level when using $R A T_{k}$.

- range $_{k}$ : an assessment of the range to reflect the appropriateness from the UE velocity perspective.

Therefore, for each application $A_{l}$, the RATs can be fully characterized in terms of a $K x M$ decision matrix $\mathbf{D}_{1}$ whose element $d_{k, m}^{l}$ denotes the performance of $R A T_{k}$ in terms of the $m$-th attribute:

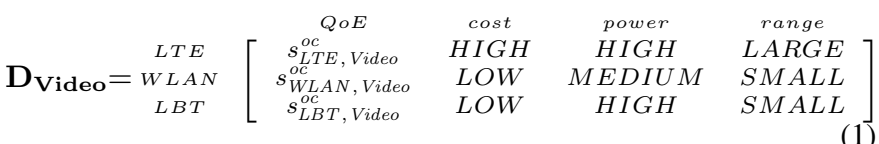

$$
\mathbf{D}_{\mathbf{F T P}}=\begin{gathered}
\text { LTE } \\
\text { WLAN } \\
L B T
\end{gathered}\left[\begin{array}{cccc}
\text { QoS } & \text { cost } & \text { power } & \text { range } \\
H I G H & \text { HIGH } & \text { HIGH } & \text { LARGE } \\
H I G H & \text { LOW } & \text { MEDIUM } & \text { SMALL } \\
H I G H & \text { LOW } & \text { HIGH } & \text { SMALL }
\end{array}\right]
$$

Note that, compared to licensed access (i.e., LTE), unlicensed RATs (i.e., WLAN and LBT) are qualified as cheaper and smaller for both applications. In turn, the LTE-based options (i.e., LTE and LBT) are judged as more power consuming than WLAN. Note that the out-of-context suitability level to support FTP transfer (i.e., $s_{k, F T P}^{o c}$ ) is always set to HIGH to reflect the loose QoS requirements.

To adjust the relative importance of the various attributes, a vector $\mathbf{w}_{\mathbf{l}}^{\mathbf{s}}$ of $M$ weights $\left(\left\{w_{l, m}^{s}\right\}_{1 \leq m \leq M}\right)$ is introduced for each $l$-th application and $s$-th subscription profile:

$$
\mathbf{w}_{\mathbf{V i d e o}}^{\mathbf{B}}=\mathbf{w}_{\mathbf{F T P}}^{\mathbf{B}}=\left[\begin{array}{c}
H I G H \\
H I G H \\
L O W \\
L O W
\end{array}\right]
$$

$$
\mathbf{w}_{\mathbf{V i d e o}}^{\mathbf{G}}=\mathbf{w}_{\mathbf{F T P}}^{\mathbf{G}}=\left[\begin{array}{c}
H I G H \\
L O W \\
L O W \\
L O W
\end{array}\right]
$$

where $\mathrm{B}$ and $\mathrm{G}$ stand for the Bronze and Gold subscription profiles, respectively.

Note that, for both applications, the cost attribute is more relevant for the Bronze user (i.e., $w_{l, \text { cost }}^{B}=H I G H$ ). In turn, the velocity and power attributes are not initially considered for the sake of simplicity (i.e., $w_{l, \text { power }}^{s}=w_{l, \text { range }}^{s}=L O W$ ).

Finally, the vector $\mathbf{s}_{\mathbf{l}}^{\mathbf{i c}, \mathbf{s}}$ of in-context suitability levels $\left(\left\{s_{k, l}^{i c, s}\right\}_{k \in\{1, \ldots, K\}}\right)$ is obtained by combining the attributes and weights as follows:

$$
\mathbf{s}_{\mathbf{l}}^{\mathbf{i c}, \mathbf{s}}=\left[\begin{array}{c}
s_{1, l}^{i c, s} \\
\vdots \\
s_{k, l}^{i c, s} \\
\vdots \\
s_{K, l}^{i c, s}
\end{array}\right]=\overline{\mathbf{D}_{\mathbf{l}}} \cdot \mathbf{w}_{\mathbf{l}}^{\mathbf{s}}
$$

where $\overline{\mathbf{D}_{1}}$ is the matrix of normalized attributes $\overline{d_{k, m}^{l}}$ that are calculated as $\overline{d_{k, m}^{l}}=d_{k, m}^{l} / \max _{k}\left(d_{k, m}^{l}\right)$ for benefit attributes (i.e., QoS/QoE and range) and $\overline{d_{k, m}^{l}}=\min _{k}\left(d_{k, m}^{l}\right) / d_{k, m}^{l}$ for cost attributes (i.e., cost and power).

\section{Decision-making}

Based on the previous section, the RAT that maximises the in-context suitability level is selected for the $l$-th application and $s$-th subscription profile:

$$
k^{*}(l, s)=\arg \max _{k \in\{1, \ldots, K\}}\left(s_{k, l}^{i c, s}\right)
$$

To track the variability in the various attributes (e.g., radio conditions and contextual information), the CM implements the following functionalities based on the above criterion:

- Spectrum selection (SS): the best RAT is selected at the time of establishing each of the considered applications.

- Spectrum mobility (SM): a handover (HO) to the best RAT is performed during sessions. Two types of HO are considered, namely event-triggered emergency HOs (i.e., whenever any of the application requirements is not met) and periodic comfort HOs triggered each $\Delta T$. In both cases, if a better $R A T_{k^{*}}$ is identified (i.e., $\left.s_{k^{*}, l}^{i c, s}>s_{\text {serving, } l}^{i c, s}\right)$, the UE is reconfigured to use it.

\section{Simulation RESUlTS}

To get insight into the relevance of the proposed approach in performing a context-aware operation, a set of systemlevel simulations have been performed using the NS-3 simulator [14].

\section{A. Considered environment}

- To model ultra-dense deployments, a single LTE macrocell overlaid by a set of buildings is considered. Each building is structured according to the dual-stripe layout [15] i.e., as two stripes of rooms with a corridor in-between, which corresponds in practice to e.g., the set of stores inside a shopping mall. The various propagation losses (i.e., indoor-indoor, outdoor-outdoor, 


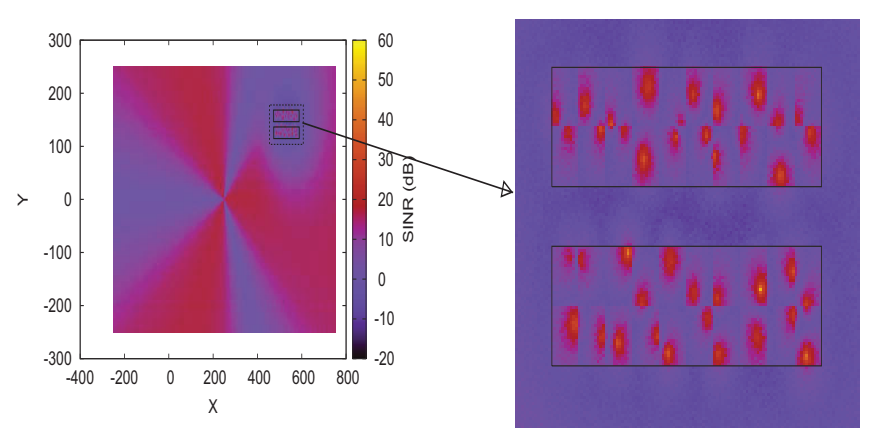

Fig. 6. Illustrative example of SINR map, licensed band

indoor-to-outdoor and vice versa) are modeled using the hybrid building model that combines several well known propagation loss models [16].

- A set of small-cells are dropped randomly inside each building and allowed to operate in both licensed and unlicensed bands. The licensed band is shared with the macro-cell according to a co-channel deployment, while the unlicensed band is shared with a WLAN 802.11n AP placed inside the same room. As an illustrative example, Fig. 6 describes the signal-to-interference-andnoise-ratio (SINR) map obtained in the licensed band when a building composed of two 20-room stripes is considered with one small-cell placed in each room.

- The full list of physical (i.e., LTE, WLAN and LBT) parameters to access licensed/unlicensed bands is that of the outdoor scenario described in the Annex A of [4].

\section{B. Traffic model}

The $L=2$ applications considered in Section. II-A are modelled as follows:

- Interactive video: During an interactive (e.g., live streaming) session, the UE receives a given video sequence from a remote host over a UDP transport session. The associated set of requirements is characterized in terms of a maximum end-to-end delay of $D_{\max }=100 \mathrm{~ms}$ and frame loss ratio of $L_{\max }=0.1 \%$. In this respect, the video receiver accepts only in-sequence frames whose end-toend delay does not exceed $D_{\max }$. Any other frame is dropped with no subsequent retransmission. An approach to simulate the actual video transmission and assess the perceived QoE will be presented in the next sub-section.

- FTP transfer: an ON/OFF model is used to model FTP download sessions (i.e., ON periods) and the inactivity intervals in-between (i.e., OFF periods). Both ON/OFF periods are exponentially distributed with rate $\lambda$. To control the load generated by FTP sessions, each ON period is assumed to consume a percentage $\rho$ of the capacity of the in-use radio link with loose QoS requirements.

\section{Evaluation of video $Q o E$}

Given the high cost incurred by subjective tests, this section proposes to perform an objective assessment of the video QoE. In this respect, the Evalvid framework [17], whose simplified architecture is described in Fig. 7, has been integrated.

On the sender side, a digital video sequence is encoded, packetised and transmitted over a simulated network. On the receiver side, a play-out buffer is optionally used for

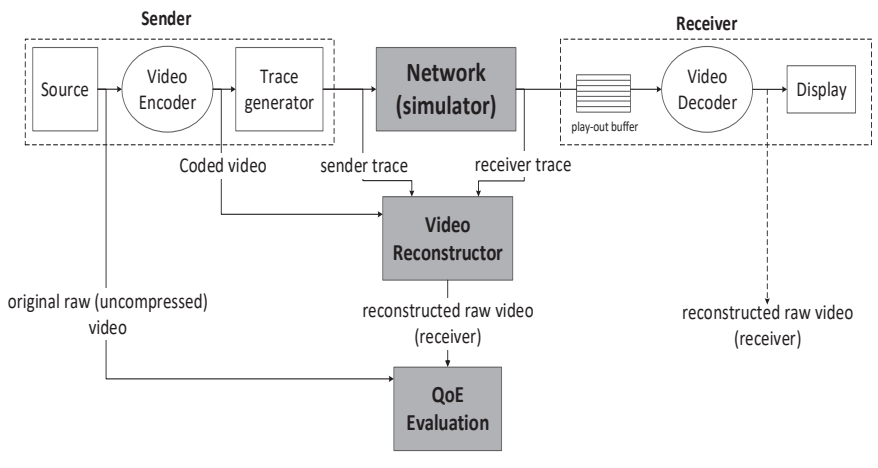

Fig. 7. A simplified architecture of the Evalvid framework

jitter reduction before the received sequence is decoded and displayed. Both the sender and receiver keep track of the time-stamp and type of each sent/received packet in separate trace files. These trace files are combined with the original encoded video to reconstruct the uncompressed raw video as it would be perceived by the receiver. Based on a comparison between the original and reconstructed raw sequences, the QoE is evaluated based on the following metrics:

- The peak signal to noise ratio (PSNR) which has been extensively used in the literature for its simplicity. It provides a measure of the similarity between a distorted video sequence and its original counterpart based on a frame-by-frame comparison [17].

- The structural similarity (SSIM) index which focuses on the structural information loss to which the human visual system is strongly sensitive. It computes the mean, variance and covariance of small patches inside each frame and combines them into a distortion map [18].

To reflect the current trend in high-end video entertainment, the popular Big Buck Bunny animated video (e.g., 16+ million views on Youtube) has been selected as test sequence. Its uncompressed raw stream was downloaded from [19] and encoded with H264 (Main Profile, L4) at 1080p @24fps, which generates a variable bit-rate with an average of $2.5 \mathrm{Mbps}$.

\section{Benchmarking}

To assess the efficiency of the proposed framework in selecting the best RAT, the fuzzy MADM strategy developed in Section. III is applied in the following scenarios:

- Offloading: LTE small-cells are allowed to operate only in licensed bands. To exploit unlicensed bands, part of the traffic may be offloaded to WLAN.

- Sharing: Small-cells can operate in licensed and unlicensed modes. The unlicensed band is shared with the existing WLAN based on the LBT procedure.

- Offloading+Sharing: This scenario combines the previous two options. Therefore, unlicensed bands can be jointly exploited through offloading and sharing.

Additionally, to benchmark the performance of the proposed approach, the following baseline is considered:

- Fixed: A traditional scheme that always assigns delaysensitive traffic (i.e., interactive video) to licensed bands (i.e., LTE) and delay-tolerant traffic (i.e., FTP transfer) to unlicensed bands (i.e., WLAN or LBT). 


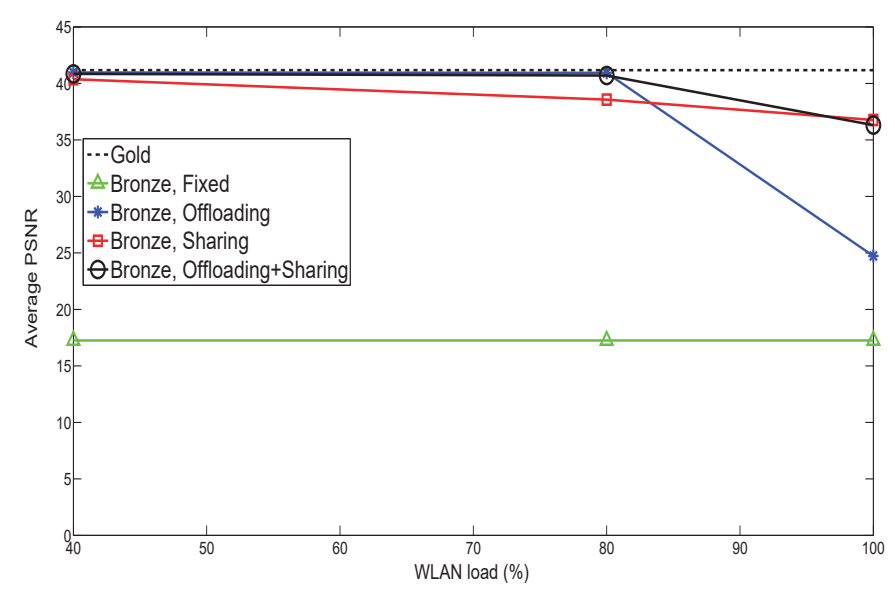

Fig. 8. Impact of WLAN load on the perceived QoE in terms of PSNR

\section{E. Initial assumptions}

To provide a proof of concept of the proposed approach, the following assumptions are initially considered:

- A single-room scenario of the dual-stripe layout described in Section. IV-A is initially considered.

- To model the existing load on unlicensed bands, a single FTP session with $\frac{1}{\lambda}=2 s$ is initially established over WLAN. Therefore, the percentage of used capacity defined in Section. IV-B (i.e., $\rho$ ) can be used as an indicator of the existing load on WLAN.

- Two Bronze and Gold video sessions are established in the same room. The Bronze subscription profile is associated with a limited balance of 500Mbits.

\section{F. Performance evaluation}

This section evaluates the effectiveness of the proposed fuzzy MADM strategy in performing a context-aware exploitation of all available bands in the considered environment. To this end, the QoE metrics introduced in Section. IV-C are evaluated.

Fig. 8 shows the average PSNR perceived during video sessions as a function of the WLAN load when comfort HOs are triggered each $\Delta T=200 \mathrm{~ms}$. The various variants represent the performance of the proposed strategy in each of the scenarios described in Section. IV-D together with the baseline Fixed. Note that the performance achieved by the Bronze user is shown separately for each variant. In turn, the performance of the Gold user is shown only once as it does not depend on the used variant. Finally, it has been checked that the QoE performance in terms of SSIM exhibits a similar behavior and is therefore omitted for the sake of brevity.

The results first show that the performance of the Gold user remains unchanged for all considered variants. This is because according to the strategy developed in Section. III, the cost attribute is irrelevant for the Gold subscription profile, and as a result, the paid well-regulated LTE is always used. Note that the baseline Fixed achieves an equal performance as it statically assigns interactive video sessions to LTE.

Next, the QoE perceived by the Bronze user is analysed. To better understand the relative performance achieved by each scheme, Fig. 9(a) and Fig. 9(b) plot the evolution of the instantaneous (i.e., per frame) PSNR perceived by the Bronze user when the WLAN load is low (i.e., 40\%) and high (i.e., $100 \%$ ), respectively.
The first key observation in Fig. 8 is that the improvement introduced by the unlicensed access is significant (i.e., ranging from $100 \%$ to $135 \%$ ) for all considered options (i.e., Offloading, Sharing, Offloading +Sharing). This is because the limited balance of the Bronze user is not enough to deliver the whole video stream over the paid LTE access. As a consequence, when only the licensed band is exploited (i.e., Fixed), the video session shortly gets dropped after the balance is depleted at frame ID=3300 as highlighted in Fig. 9(a). The additional exploitation of the unlicensed band saves some of the valuable balance units and helps to maintain the video session till the end, which significantly improves the average PSNR.

When comparing the various options to exploit unlicensed bands, it can be observed in Fig. 8 that the best option strongly depends on the existing load on WLAN. At low loads, the unused WLAN capacity is enough to accommodate the video traffic, which justifies the good performance maintained by Offloading in Fig. 9(a). On the contrary, contending with WLAN for the unlicensed band results in some collisions as identified by the sharp PSNR drops experienced by Sharing in Fig. 9(a). To assess the impact of these degradations on the end-user perception, Fig. 10 shows the actual frame perceived by the Bronze user at the position indicated in Fig. 9(a) (i.e., frame ID=5900). It can be seen that unlike the other schemes, the reconstructed frame by Sharing is strongly degraded. As the load increases, Offloading is forced to often use the licensed band, which results in an "out-of balance" drop at frame ID=7320 as highlighted in Fig. 9(b). In turn, Sharing manages to maintain the video session till the end, which justifies the achieved gain in Fig. 8 with respect to Offloading for high loads. When offloading and sharing are jointly considered (i.e., Offloading +Sharing), the combined approach efficiently switches between both options depending on the operating conditions as can be observed in Fig. 8.

In summary, the proposed fuzzy MADM strategy enables to select the best RAT depending on the context at hand. For Gold users, it exclusively exploits the well-regulated licensed band as the cost is not of concern. For Bronze users, it additionally exploits unlicensed bands depending on the operating conditions of the various RATs. It mainly performs Offloading when the existing load on WLAN is low, while switches to Sharing when the WLAN gets saturated. This results in a significant improvement of the perceived QoE compared to the out-of-context baseline scheme.

\section{CONCLUSIONS AND FUTURE DIRECTIONS}

This paper proposes a novel context-aware user-driven strategy to efficiently exploit all available bands and licensing regimes in ultra-dense environments without any prior knowledge about each combination. It relies first on fuzzy logic to estimate the suitability of each available RAT to support various applications subject to the uncertainty level associated with UEs. Then, a fuzzy MADM approach is developed to combine these estimates with the heterogenous components of the context to assess the in-context suitability levels. Based on this metric, a spectrum management strategy combining two spectrum selection (SS) and spectrum mobility (SM) functionalities is developed to select the best RAT in a given context. As an illustrative use case, the proposed strategy is applied to support interactive video sessions for a set of Bronze (i.e., limited-balance) and Gold (i.e., flat-rate) subscription profiles. The results reveal that the proposed approach always 


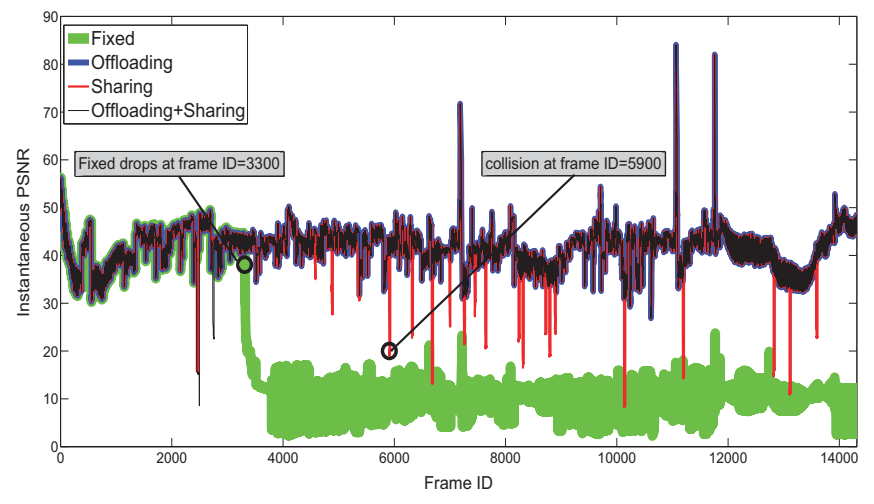

(a) WLAN load $=40 \%$

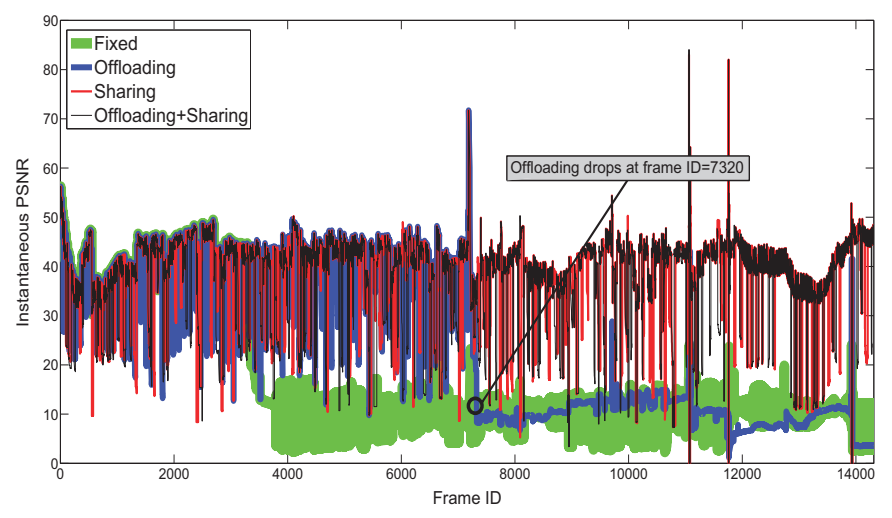

(b) WLAN load $=100 \%$

Fig. 9. Evolution of the instantaneous PSNR of the Bronze user

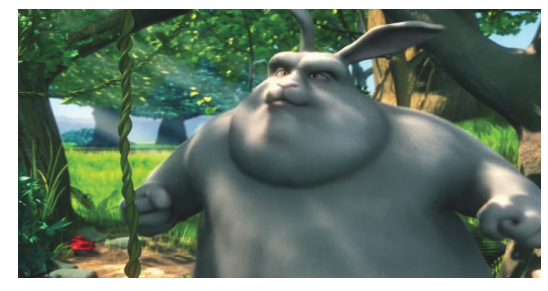

(a) Offloading

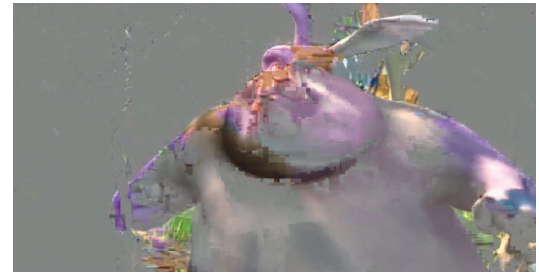

(b) Sharing

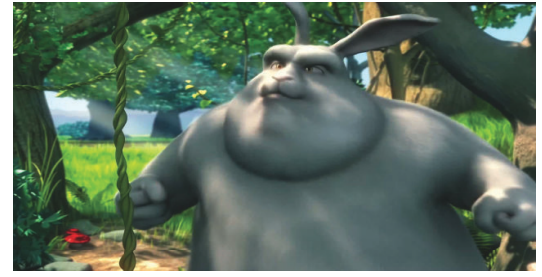

(c) Offloading+Sharing

Fig. 10. The actual frame perceived by the Bronze user at frame ID $=5900$, WLAN load $=40 \%$

assigns Gold users to the well-regulated licensed band, while switches Bronze users between licensed and unlicensed bands depending on the operating conditions of each RAT. This results in a significant improvement (i.e., 100\% to 135\%) of the achieved QoE compared to a traditional scheme that exploits only licensed bands. Then, a comparative study is conducted between the two available options to exploit unlicensed bands, namely Offloading and Sharing. The results show that the best option strongly depends on the existing load on WLAN. At low loads, Sharing suffers from few collisions, while Offloading runs "out-of balance" at high loads. Therefore, a combined approach is proposed to efficiently switch between both options, which achieves the best QoE for all considered loads.

As part of future work, it is intended to develop more advanced context-aware (e.g., energy-aware) strategies and implement the proposed approach in a real-word environment.

\section{ACKNOWLEDGEMENTS}

The authors would like to gratefully acknowledge the support of BT through the University of Surrey 5G Innovation Centre (http://www.surrey.ac.uk/5gic), and the EU funded H2020 5G-PPP project SPEED-5G under the grant agreement no. 671705 .

\section{REFERENCES}

[1] J. G. Andrews, H. Claussen, M. Dohler, S. Rangan, and M. C. Reed, "Femtocells: Past, present, and future," IEEE Journal on Selected Areas in Communications, vol. 30, no. 3, pp. 497-508, April 2012.

[2] K. Lee, J. Lee, Y. Yi, I. Rhee, and S. Chong, "Mobile data offloading: How much can WiFi deliver?" IEEE/ACM Transactions on Networking, vol. 21, no. 2, pp. 536-550, April 2013.

[3] Qualcomm, LTE-U coexistence mechanism. [Online]. Available: http://www.lteuforum.org/uploads/3/5/6/8/3568127/lteu_ coexistence_mechansim_qualcomm_may_28_2015.pdf

[4] 3GPP, "Study on licensed-assisted access to unlicensed spectrum, (Release 12)," Tech. Rep. 36.889-V13.0.0, June 2015.
[5] Qualcomm news, introducing muLTEfire: LTElike performance with Wi-Fi-like simplicity. [Online]. Available: https://www.qualcomm.com/news/onq/2015/06/11/ introducing-multefire-lteperformance-wi-fi-simplicity

[6] A. R. Elsherif, W. P. Chen, A. Ito, and Z. Ding, "Adaptive small cell access of licensed and unlicensed bands," in 2013 IEEE International Conference on Communications (ICC), June 2013, pp. 6327-6332.

[7] Q. Chen, G. Yu, R. Yin, A. Maaref, G. Y. Li, and A. Huang, "Energy efficiency optimization in licensed-assisted access," IEEE Journal on Selected Areas in Communications, vol. 34, no. 4, pp. 723-734, April 2016.

[8] Q. Chen, G. Yu, A. Maaref, G. Y. Li, and A. Huang, "Rethinking mobile data offloading for LTE in unlicensed spectrum," IEEE Transactions on Wireless Communications, vol. 15, no. 7, pp. 4987-5000, July 2016.

[9] F. Bouali, K. Moessner, and M. Fitch, "A Context-aware User-driven Framework for Network Selection in 5G multi-RAT Environments," in IEEE 84th Vehicular Technology Conference (VTC Fall), September 2016.

[10] Open Mobile Alliance, "OMA Device Management Protocol," Tech. Rep. version 1.2.1, June 2008.

[11] L. Zadeh, "Fuzzy sets," Information and Control, vol. 8, no. 3, pp. 338 $-353,1965$.

[12] Intel et al., "WF on contention window adaptation based on HARQ ACK/NACK feedback," 3GPP TSG RAN WG1 \#82bis, Tech. Rep. R1156332, October 2015

[13] C.-L. Hwang and K. Yoon, Multiple Attribute Decision Making: Methods and Applications A State-of-the-Art Survey. Springer Berlin Heidelberg, 1981, ch. Methods for Multiple Attribute Decision Making, pp. 58-191.

[14] The network simulator-3 (NS-3). [Online]. Available: https://www. nsnam.org/

[15] 3GPP, "TSG RAN WG4 Meeting 51: Simulation assumptions and parameters for FDD HeNB RF requirements," Tech. Rep. R4-092042, May 2009.

[16] "NS-3 Model Library," Tech. Rep. Release NS-3.25, 2016. [Online]. Available: https://www.nsnam.org/docs/release/3.25/models/ ns-3-model-library.pdf

[17] J. Klaue, B. Rathke, and A. Wolisz, EvalVid - A Framework for Video Transmission and Quality Evaluation. Springer Berlin Heidelberg, 2003, pp. 255-272.

[18] Z. Wang, A. C. Bovik, H. R. Sheikh, and E. P. Simoncelli, "Image quality assessment: from error visibility to structural similarity," IEEE Transactions on Image Processing, vol. 13, no. 4, pp. 600-612, April 2004.

[19] The Big Buck Bunny. [Online]. Available: https://peach.blender.org/ 